\title{
Evolución de la artrodesis cervical postdiscectomía: injerto óseo, placa, caja intersomática y placa-caja
}

\author{
J.M. González-Darder
}

Servicio de Neurocirugía. Hospital General de Castellón

\section{Resumen}

Se revisan las técnicas de artrodesis cervical anterior postdiscectomía con injerto óseo, placa cervical, caja intersomática y placa-caja, discutiendo las ventajas mecánicas que aporta cada sistema. La alternativa a la artrodesis es la discectomía simple o la artroplastia con prótesis discal. Los sistemas placa-caja integrados pueden considerase como el resultado final de la evolución de los conceptos de artrodesis cervical. Se describen detalladamente los sistemas placa-caja actualmente disponibles.

PALABRAS CLAVE: Fusión cervical. Placa cervical anterior. Caja intersomática. Sistemas placa-caja.

Development of the anterior cervical postdiscectomy arthrodesis: bone graft, plate, intersomatic cage and plate-cage

\section{Summary}

The surgical techniques for anterior cervical postdiscectomy arthrodesis are reviewed, including bone graft, cervical plates, intervertebral cages and plate-cage systems. The biomechanical advantages of each method are discussed. An alternative to arthrodesis are simple discectomy and arthroplastia with disc prosthesis. Integrated plate-cage systems are the final result in the evolution of the cervical arthrodesis developments. The available plate-cage systems are described.

KEY WORDS: Cervical fusion. Anterior cervical plate. Intervertebral cage. Plate-cage systems.

\section{Introducción}

La discectomía cervical por vía anterior es una maniobra descompresiva de los elementos neurológicos $\mathrm{y}$, al

Recibido: 28-02-05. Aceptado: 06-04-05 producir necesariamente la extirpación de determinados elementos estructurales del raquis cervical que colaboran en la estabilidad del mismo como son el ligamento vertebral común anterior, disco y, eventualmente, el ligamento vertebral común posterior, es un acto quirúrgico que puede incrementar o producir inestabilidad. En estudios biomecánicos in vitro, Schulte et $\mathrm{al}^{33}$ observaron que la discectomía C5C6 aumenta de forma altamente significativa la movilidad del segmento en flexión (66.6\%), extensión $(69.5 \%)$, inclinación lateral $(41.3 \%)$ y rotación axial (37.9\%). Con la artrodesis postdiscectomía se pretende restablecer la estructura de la columna recuperando su rigidez y estabilidad para evitar movimientos anómalos que produzcan o hagan progresar la deformidad o que produzcan o mantengan el dolor secundario a la misma.

Los sistemas de artrodesis postdiscectomía cervical tratan de alcanzar la estabilidad del segmento intervenido gracias, inicialmente, al soporte mecánico de la osteosíntesis y, tardíamente, por la fusión ósea; adicionalmente se pretende mantener o, en su caso, restituir la altura del espacio intervertebral y, de esta manera, el diámetro de los agujeros de conjunción por donde salen los nervios raquídeos cervicales; $y$, finalmente, la artrodesis trata de mantener o, en su caso, restituir la alineación normal del segmento intervenido. La estabilidad del segmento artrodesado también favorecería la reabsorción de los osteofitos posteriores no resecados tras la discectomía. En la mielopatía cervical la artrodesis eliminaría el factor dinámico involucrado en su patogénesis y favorecería la tensión de los ligamentos amarillos que improntan sobre la cara posterior de la médula espinal (Figura 1).

Todos estos objetivos se deben obtener manteniendo la seguridad de los elementos osteoarticulares y neurológicos implicados en el área anatómica, con una técnica quirúrgica lo más sencilla posible que minimice las probabilidades de complicaciones y con un diseño del implante que asegure su integridad a largo plazo. Finalmente, debe considerarse el impacto económico que el sistema de fijación repercute sobre el coste del proceso asistencial.

Teniendo en cuenta todas estas consideraciones teóricas acerca de los requerimientos del sistema de artrodesis ideal, 
- Restituir / mantener la estabilidad del segmento.

- Restituir / mantener la separación intersomática.

- Restituir / mantener la apertura agujeros de conjunción.

- Restituir / mantener la alineación en lordosis del segmento.

- Favorecer la reabsorción de los osteofitos posteriores.

- Eliminar el factor dinámico en caso de mielopatía.

- Tensar los ligamentos amarillos en caso de mielopatía.

Figura 1. Objetivos de la artrodesis cervical postdiscectomía.

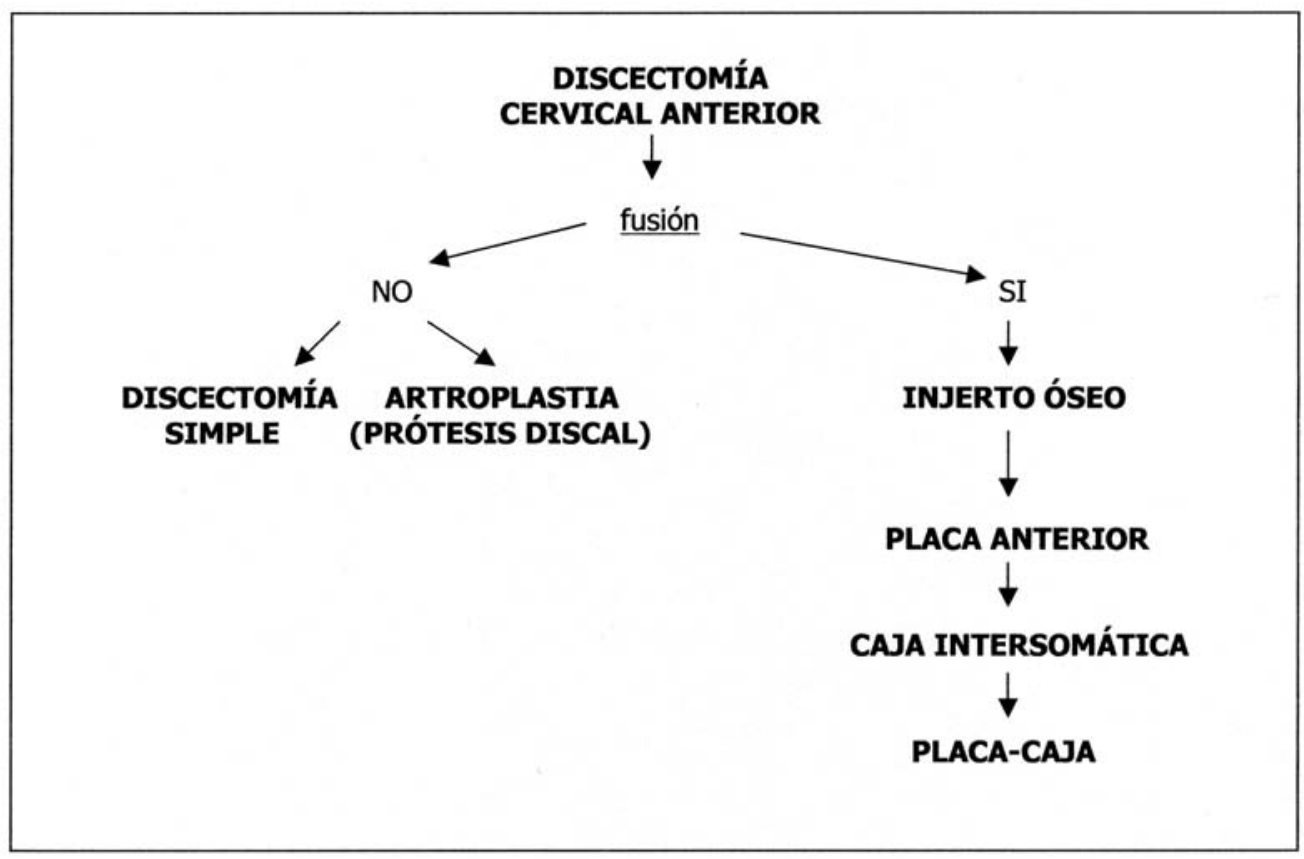

Figura 2. Evolucion de las técnicas postdiscecctomia cervical.

el concepto de fijación con placa-caja para la artrodesis cervical postdiscectomía puede considerarse como una evolución natural de la técnica de fusión postdiscectomía iniciada con el injerto óseo en los años cincuenta, seguido más tarde con la asociación de sistemas de osteosíntesis con placas anteriores y cajas intersomáticas (Figura 2). El objetivo del presente trabajo es revisar esta evolución, indicando en cada caso la justificación y las mejoras obtenidas en cada paso.

\section{Injerto óseo}

La artrodesis con injerto de hueso se obtiene gracias al paso de trabéculas óseas entre los elementos a artrodesar.
El papel básico del injerto de hueso es la inducción de la osteogénesis ósea y la osteoconducción, pero el implante de hueso tiene a su vez otros diferentes papeles, ya que también proporciona estabilidad inmediata y actúa como espaciador y en la distribución de cargas 7 . Cuando un injerto de hueso se somete a cargas de compresión se induce la osteogénesis ósea, mientras que cuando es sometido a distracción se inhibe la osteogénesis. Este es el principio de Wolf que, junto a otros aspectos básicos como la calidad del hueso, superficie de contacto y los factores biológicos sistémicos involucrados en transcurso de la osificación, han de ser conocidos para entender el proceso de artrodesis por fusión ósea.

La artrodesis postdiscectomía cervical se realizó ini- 
cialmente con injertos de hueso. El hueso autólogo tricortical de cresta iliaca reúne tantas ventajas para su uso en esta indicación concreta que se continúa utilizando desde el principio para esta finalidad. La fusión cervical anterior con injerto óseo fue realizada originariamente por Bailey y Badgley ${ }^{4}$ en los años cincuenta y las bases técnicas todavía vigentes ahora se establecieron muy inicialmente por Cloward ${ }^{6}$ y Smith y Robinson ${ }^{34}$ para el injerto cilíndrico y el rectangular. Con posterioridad se han usado una ingente variedad de implantes de hueso autólogo, homólogo o heterólogo, así como otros materiales. Estudios "in vitro" del segmento $\mathrm{C} 5 \mathrm{C} 6$, tras una discectomía, demostraron que la colocación de un injerto óseo intersomático disminuye significativamente la movilidad del segmento en extensión, inclinación lateral y rotación en casi el 50\% $y$, en menor grado, la flexión ${ }^{33}$. En un estudio propio el injerto intersomático tricortical de cresta iliaca autóloga aumenta de forma global la altura relativa del disco respecto a los cuerpos vecinos del $15.3 \%$ en el preoperatorio hasta el $16.9 \%$ entre los 6-9 meses del postoperatorio, sin apreciarse cambios en el ángulo del segmento intervenido, mientras que la artrodesis ósea en los estudios radiológicos dinámicos sólo se alcanzaba en el $65 \%$ de los casos ${ }^{13}$. Un grupo de nuestro medio, con amplia experiencia con la técnica de Cloward, obtiene una tasa de fusión del 90.4\% ${ }^{22}$.

Dados los problemas inmunológicos y de seguridad biológica relacionados con los implantes de hueso homólogo o heterólogo, en la actualidad se ha vuelto al uso del injerto autólogo de cresta iliaca. Dejando aparte las complicaciones asociadas a la toma de injerto, a veces exageradas para justificar el empleo de otros materiales comerciales, el problema más importante del injerto desde el punto de vista mecánico es la pérdida de altura por asentamiento del mismo tras su implante, siendo la máxima expresión del fracaso del injerto el colapso, rotura o extrusión y la pseudoartrosis. Con relación a este tema, el primer aspecto a considerar es la selección de la altura ideal a la hora de tallar el injerto intersomático. An et al. ${ }^{1}$ estudiaron este problema recomendando que para una altura discal de 3.5$6.0 \mathrm{~mm}$ el espesor mas adecuado son $2 \mathrm{~mm}$ más que la altura basal preoperatoria. Esta altura sería la idónea para mantener la apertura del agujero de conjunción, aunque el diámetro del foramen también se ve influido por la curvatura cervical, ya que la lordosis cervical exagerada lo reduciría. Desgraciadamente, el injerto óseo pierde sistemáticamente altura con el paso del tiempo. En nuestra experiencia clínica, no publicada, el injerto óseo intersomático de cresta iliaca pierde aproximadamente un $20 \%$ de altura en controles radiológicos realizados a los seis meses, lo que reduciría los requerimientos biomecánicos del injerto favoreciendo la cifosis. Sin embargo, está ampliamente aceptado que un injerto de excesiva altura que pretenda compensar el subsiguiente asentamiento conduce a la cervicalgia postoperatoria por distensión exagerada de los elementos ligamentosos posteriores. La asociación de una placa cervical anterior proporcionaría un complemento mecánico al injerto de altura idónea para evitar el colapso tardío.

\section{Placa cervical anterior}

Los pioneros en el empleo de una placa cervical anterior para apoyar el injerto óseo intersomático postdicectomía con el fin de mejorar la estabilidad inicial y tasa de fusión ósea fueron nuestros compatriotas Orozco y Llovet ${ }^{25}$, lo que hay que destacar como un evento de auténtica relevancia histórica. Estos autores usaron una placa convencional de osteosíntesis AO de huesos largos, poniendo en marcha el desarrollo de numerosas variantes en el diseño de las placas cervicales anteriores. La asociación de una placa cervical al injerto óseo reduce significativamente (70\%) la movilidad del segmento en flexión, extensión, inclinación lateral y rotación axial en estudios "in vitro" tras discectomía C5C6 $6^{33}$. En un estudio propio, la placa cervical anterior asociada al injerto intersomático de cresta iliaca aumenta la altura relativa del disco del $15.3 \%$ en el preoperatorio hasta el 19,8\% entre los 6-9 meses del postoperatorio, con aumento del ángulo, en lordosis, del segmento intervenido en $2.6^{\circ}$, siendo las diferencias en ambos casos significativas estadísticamente, y alcanzándose la artrodesis ósea en los estudios radiológicos dinámicos en el 100\% de los $\operatorname{casos}^{13}$.

Una interesante revisión de este tema y un intento de clasificación funcional de las placas cervicales ha sido publicada por Haid et $\mathrm{al}^{15}$. Estos autores clasifican las placas en un primer momento de acuerdo a la relación tornillo-placa. En los sistemas pioneros ('unrestricted backout') el tornillo no encajaba en la placa, de forma que se dirigía libremente y se recomendaba que fuera bicortical, permitiendo cierto asentamiento al sistema cuando el injerto pierde altura por la compresión. A cambio, esta compresión favorecería la osteogénesis según la ley de Wolf. La tasa de roturas y aflojamientos era alta, por lo que se desarrollaron sistemas ('restricted backout') donde el tornillo se encaja rígidamente en la placa marcando una trayectoria obligatoria para el mismo ('constrained'), siendo ahora innecesaria la fijación bicortical. La rigidez de estos sistemas iría en contra de la ley de Wolf y se dificultaría la osteogénesis. Sin embargo, estos sistemas se han desarrollado ampliamente con tasas de fusión próximas al 100\%. Una línea de evolución ha sido la invención de sistemas de bloqueo que impiden la salida del tornillo en caso de aflojamiento, lo que permite al tiempo la posibilidad de una cierta movilidad del tornillo ('semi-constrained'), bien sea a la hora de elegir la dirección adecuada en su introducción o permitiendo una cierta movilidad del tornillo con relación a la placa cuando se produce el asentamiento del injerto. 
También es importante para el cirujano implantador permanecer al corriente de los rudimentos biomecánicos de los implantes para conocer mejor las razones del diseño y estar más atento a los detalles de la técnica quirúrgica de la implantación. En este sentido se han publicado algunas revisiones sistemáticas muy ilustrativas y de fácil compren$\operatorname{sión}^{7,23,32}$.

El problema más importante de los sistemas de osteosíntesis, dejando aparte las complicaciones relacionadas con su implantación, es el fracaso mecánico del sistema en forma de rotura o aflojamiento. En general, dado que el diseño de los sistema se basa en estudios biomecánicos que aseguran la resistencia del material, asumiendo una correcta técnica y en ausencia de patología ósea local, el fracaso estructural del material se produce muchas veces tras el fracaso previo del injerto que modifica las cargas mecánicas sobre tornillos o placas y conduce a su rotura o aflojamiento ${ }^{32}$. Un sistema que evitara el asentamiento o colapso del injerto impediría un gran número de fracasos de los implantes, lo que es una de las causas que justifican la asociación de una caja intersomática a la placa cervical sustituyendo el injerto intersomático.

\section{Caja intersomática}

Con el fin de estandarizar la técnica de fusión intersomática y de eliminar la necesidad de toma de injerto, se desarrollaron las cajas intersomáticas de diferentes materiales, bien con forma cilíndrica o de paralelepípedo. Los estudios clínicos demuestran que los resultados clínicos y radiológicos son superponibles a los de la fusión obtenida con injerto óseo ${ }^{24,35,37}$. De la misma forma, diferentes estudios biomecánicos con especímenes humanos han demostrado que la caja intersomática se comporta de forma semejante al injerto tricortical de cresta iliaca ${ }^{9,18,26}$. Estos mismos estudios biomecánicos "in vitro" demuestran también, de forma sistemática, que la incorporación de una placa cervical anterior aumenta significativamente la estabilidad y reduce el rango de movimiento, tanto de los montajes con injerto de cresta como con cajas intersomáticas.

Diferentes estudios clínicos han llamado la atención acerca del asentamiento tras el implante de las cajas intersomáticas, tanto de las cilíndricas como de las rectangulares. Así Tureyen ${ }^{36}$ sobre 43 cajas cilíndricas encuentra una tasa de fusión del $98 \%$ pero con una pérdida de altura del disco del $35 \%$ y Gercek et al. ${ }^{10}$ encuentran signos radiológicos de asentamiento en 5 de 9 niveles fusionados con cajas cuadradas. Porras-Estrada et al. ${ }^{27}$ observan este fenómeno en todos sus casos y lo atribuyen a un fenómeno de acomodación del implante que, sin embargo, alcanzaba más del 20\% del resto del cuerpo vertebral en el 23\% de los casos. En estos casos la pérdida de altura puede deberse al desmoronamiento del hueso del soma vertebral o al colapso del disco. En todo caso, el asentamiento podría evitarse con la ayuda mecánica proporcionada por la asociación de una placa anterior.

Aparte del asentamiento, el gran problema de las cajas es su movimiento. Hay pocas publicaciones acerca de la extrusión anterior de las cajas cervicales y menos de las migraciones posteriores con la consecuente lesión neurológica. Para impedir la migración anterior se recomienda colocar la caja unos $2 \mathrm{~mm}$ por detrás del borde anterior del cuerpo vertebral. Para impedir la migración posterior se puede respetar el ligamento vertebral común posterior así como una porción más o menos amplia de la parte posterior del disco. Probablemente, por esta razón se aprecia con frecuencia en los estudios de imagen postoperatorios que las cajas se colocan relativamente anteriores. Sin embargo, la descompresión neurológica radical con exéresis de los fragmentos discales eventualmente extruídos y del osteolito posterior exige necesariamente la resección radical del disco y del ligamento, con exposición amplia de la duramadre, lo que se hace gracias al uso de la técnica microquirúrgica y del motor de alta revolución. Es evidente que tras esta maniobra radical puede producir temor la colocación de una caja, incluso aunque sea roscada o posea ranuras, curvas o rugosidades en las caras que contactan con los cuerpos vertebrales. Si la caja no establece un amplio contacto con los cuerpos vertebrales se reducen las posibilidades de fusión y se incrementan las de asentamiento y si se coloca anterior aumentan las posibilidades de migración anterior así como de excesiva lordosis que cierre los agujeros de conjunción. De nuevo, la asociación de una placa anterior mejora las posibilidades de la caja y, si se integra a la placa manteniendo la geometría del segmento operado, la situación biomecánica mejoraría de forma sustancial al tiempo que se imposibilita la movilización de la caja.

\section{Sistemas placa-caja}

Las observaciones anteriores indican que, de la misma forma que la biomecánica y resultados radiológicos del injerto tricortical mejoran con la placa cervical anterior, la asociación de una placa anterior a la caja mejora sus resultados al sumar las ventajas de ambos sistemas. En un estudio propio, el sistema placa-caja GD mejora significativamente los resultados obtenidos por el injerto óseo intersomático y del injerto asociado a placa cervical anterior. La placa-caja aumenta la altura relativa del disco del $16.4 \%$ en el preoperatorio hasta el $22.6 \%$ entre los 6-9 meses del postoperatorio, con mejoría de $5.9^{\circ}$ del ángulo del segmento intervenido a favor de la lordosis, alcanzándose en el 100\% de los casos la artrodesis ósea en los estudios radiológicos dinámicos. En este mismo estudio se demuestra que el empleo de la placa cervical o 
de la placa-caja incrementan significativamente el tiempo quirúrgico, aunque no las complicaciones ${ }^{13}$.

Aunque es posible usar una placa y una caja asociadas, se han diseñado sistemas integrados que permiten ajustar los requerimientos biomecánicos de cada uno de los componentes. Así, en el sistema integrado placa-caja la placa puede reducir su tamaño y es suficiente un tornillo por cuerpo vertebral. Cuando la caja se hace solidaria a la placa se impide totalmente la migración anterior o posterior de la primera, de forma que la descompresión posterior del disco puede ser tan radical como se precise. La caja puede rellenarse del material osteogénico u osteoconductor que se quiera. Un aspecto importante en el diseño de un sistema integrado placa-caja es que debe acoplarse a la geometría del segmento intervenido para, simultáneamente, recomponer la curvatura cervical fisiológica que usualmente está perdida en el preoperatorio en forma de cifosis. A continuación se exponen los sistemas placa-caja disponibles comercialmente.

Fixano Cervical Cage Plate (Fixano, Francia). Se trata de un sistema con una caja cilíndrica roscada de 8-10$12 \mathrm{~mm}$ de diámetro, que puede rellenarse de material osteogénico u osteoconductivo, que se rosca perpendicularmente a su vez sobre una placa muy elemental y que se fija a los cuerpos vertebrales con un tornillo por nivel. Los tornillos no tienen sistema de bloqueo. El sistema se ha diseñado para fijar un solo nivel, pero la placa es tan pequeña que podría disponerse oblicuamente y así alojar en un cuerpo el tornillo superior de un sistema y el inferior del superior en caso de dos niveles. No hay referencias bibliográficas acerca de resultados clínicos disponibles relativas al sistema.

Implant Cervical de Kehr (Groupe Fournitures Hospitalieres, Francia). Es un sistema caja-placa monobloque. Las cajas son cilíndricas no acuñadas, con medidas de $15 \mathrm{~mm}$ de diámetro y $5-6-7 \mathrm{~mm}$ de altura o de $17 \mathrm{~mm}$ de diámetro y $7-8-9 \mathrm{~mm}$ de altura. Estas cajas son, en realidad, un injerto de hidroxiapatita ajustado al interior de un cilindro metálico solidario a la placa. Las cajas son perpendiculares a las placas, de morfología en $\mathrm{H}$ y se fijan con dos tomillos por cuerpo vertebral. Kehr et al. ${ }^{19}$ describen los detalles del implante y su experiencia clínica en 46 casos.

Plaque-Cage PCB (Scient'x, Francia). Es un sistema placa-caja monobloque (figura 3). Las cajas están abiertas por arriba y abajo y miden $12 \mathrm{x} 17 \mathrm{~mm}$, con base lineal y borde superior redondeado y de $5.5-7 \mathrm{~mm}$ de altura. Perpendicular a la placa hay en su cara anterior una placa muy elemental de $26-28 \mathrm{~mm}$ de altura, en realidad dos aletas una a cada lado, donde se colocan un tornillo para cada cuerpo vertebral. Los tornillos miden $4 \mathrm{~mm}$ de diámetro y 14-16$18 \mathrm{~mm}$ de longitud, careciendo de sistema de bloqueo. Al quedar las aletas una a cada lado, es posible ir colocando sistemas en niveles sucesivos, quedando en este caso dos
2006; 17: 140-147

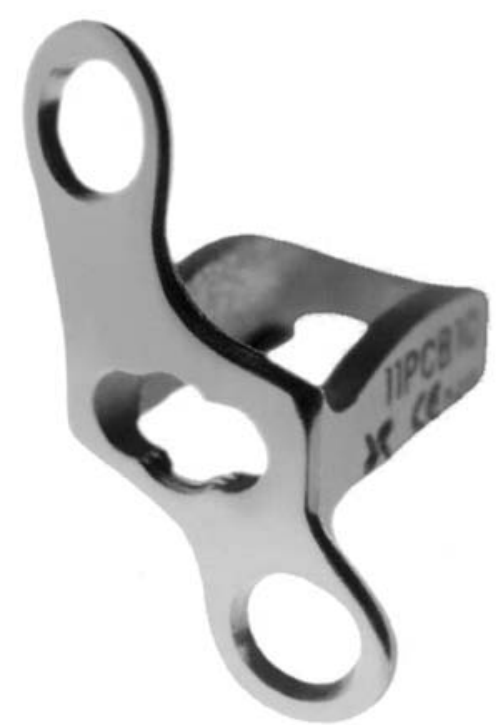

Figura 3. Placa-caja PCB (reproducida con permiso de Scient'x, Francia).

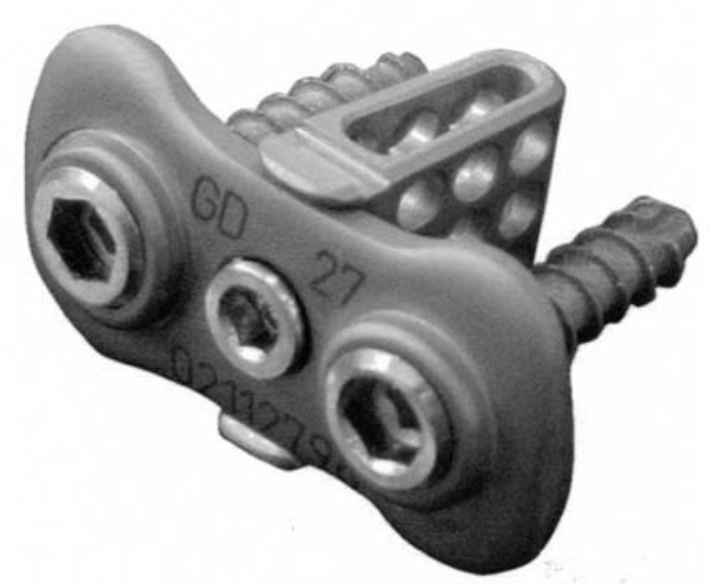

Figura 4. Placa-caja GD (reproducida con permiso de Industrias Quirúrgicas de Levante, España).

tornillos por cuerpo. La caja se rellena de material una vez colocada, ya que tiene al efecto una abertura anterior. En la literatura hay varios trabajos que recogen experiencias clínicas con este sistema, publicadas por Hwang et al. ${ }^{16}$ sobre 16 casos, Ma et al. ${ }^{21}$ sobre 22 casos y Samandouras et al. ${ }^{29}$ sobre 29 casos.

Placa-caja GD (Industrias Quirúrgicas de Levante, IQL-Biomet Merck, España). Se trata de un sistema con los dos componentes, placa y caja, aislados y que se acoplan gracias a un tornillo, lo que le confiere versatilidad (figura 4). Las placas permiten alojar una o dos cajas en diferentes posiciones y tienen longitudes variables entre 27 y $53 \mathrm{~mm}$. Las cajas son cuadradas y miden $14 \times 14 \mathrm{~mm}$, 
estando abiertas por los lados para permitir la carga previamente a la colocación del implante, mientras que sus caras superior e inferior están perforadas. Las cajas están discretamente acuñadas, miden 5-6-7-8mm de altura máxima y al atornillarse a la placa lo hacen con un ángulo de $10^{\circ}$ con el fin de acoplarse a la geometría del raquis cervical. En la literatura existen algunas publicaciones relativas a los estudios biomecánicos y clínicos preliminares del sistema ${ }^{12,13}$.

\section{Alternativas a la artrodesis intersomática}

Las alternativas a la artrodesis intersomática postdiscectomía son la discectomía simple o, más recientemente, la artroplastia con implante de un disco artificial.

Discectomía simple. Algunos autores han defendido que el empleo de los sistemas de artrodesis incrementa el tiempo operatorio y las complicaciones sin aportar ventajas adicionales a la discectomía simple. Curiosamente la discectomía simple se sigue de fusión ósea radiológica en un alto porcentaje de casos, llegando al $100 \%$ a los cuatro años en la experiencia de Savolainen et al. ${ }^{31}$, y a los 14 meses en la de Samartzis et al. ${ }^{30}$, aunque baja al $70 \%$ en el estudio de Dowd y Wirth ${ }^{8}$. Hay, sin embargo una importante tendencia a la cifosis, que se produce en el $62.5 \%$ de los pacientes del estudio de Savolainen et al. ${ }^{31} \mathrm{y}$ en el $33 \%$ de los pacientes intervenidos por Laing et al. ${ }^{20}$, quienes refieren además la pérdida de la lordosis cervical en el 55\% de los que la tenían en el preoperatorio.

En una revisión crítica de la literatura publicada sobre los procedimientos postdiscectomía cervical, Jacobs et al. ${ }^{17}$ no son capaces de extraer conclusiones válidas por la poca calidad metodológica de los trabajos disponibles, aunque apuntan que la discectomía aislada reduce el tiempo quirúrgico, de hospitalización y hasta la reincorporación laboral, mientras que no hay diferencias en los resultados clínicos sobre el dolor o tasas de fusión. Sin embargo, aunque no hay evidencia científica de peso sobre las ventajas del uso de la fusión postdiscectomía frente a la discectomía simple, es evidente que la opinión aceptada apoya el uso rutinario de la primera tras la discectomía cervical y estudios epidemiológicos realizados en Estados Unidos muestran un incremento de todas las intervenciones cervicales, incluyendo las fusiones, durante los años ochenta y la estabilización de los procedimientos durante los años noventa, pero continuando el incremento porcentual de los procedimientos de fusión ${ }^{2,5}$.

Prótesis de disco. La justificación del empleo de las prótesis discales se fundamenta, más que en mantenimiento de la movilidad del segmento intervenido, en evitar la degeneración discal adyacente al segmento artrodesado. Efectivamente, estudios a largo plazo indican que hasta en el $25 \%$ de los pacientes artrodesados tras discectomía discal desarrollan a los 10 años una discopatía cervical en los segmentos adyacentes al fusionado, y que alrededor de un $10 \%$ de ellos requieren una nueva cirugía $a^{3,22}$. Una explicación a este hecho es el aumento del estrés mecánico que sufren los segmentos vecinos al fusionado, aunque una hipótesis alternativa sería que estos discos no hacen más que seguir la historia natural de la discopatía cervical que había obligado a la primera cirugía discal y fusión subsiguiente. Por el contrario, otros estudios cuestionan estos resultados, al no encontrar diferencias en el postoperatorio en la movilidad de los segmento vecinos al fusionado ${ }^{28}$. Este es un punto muy importante, ya que la degeneración de los segmentos adyacentes es la crítica más importante en contra de la artrodesis postdiscectomía y la más sólida argumentación para el desarrollo de prótesis discales cuyo objetivo es mantener la movilidad del segmento tras las discectomía.

Los estudios clínicos realizados indican que la prótesis discal proporciona resultados clínicos semejantes a los de la discectomía con fusión, pero conservando la movilidad del segmento intervenido ${ }^{11}$. Sin embargo, los estudios actuales recomiendan un seguimiento de al menos 5 años para asegurar que la prótesis mantiene su competencia mecánica y que se protege a los discos vecinos de la degeneración.

\section{Conclusiones}

El objetivo del trabajo ha sido realizar una revisión de los fundamentos de la artrodesis anterior postdiscectomía cervical. Al ser considerada la discectomía una maniobra desestabilizadora se propuso inicialmente la artrodesis con injerto óseo, siendo los resultados radiológicos y mecánicos mejorados con la asociación de una placa cervical anterior o uso de cajas intersomáticas. El sistema placacaja integrado es la consecuencia final de estas mejoras. Sin embargo, todavía persisten dudas acerca de la necesidad de fusión postdiscectomía y algunos autores continúan recomendando la discectomía simple mientras otros implantan sistemas de artroplastia experimentales. Todos estos sistemas no producen cambios sustanciales en los resultados clínicos y el estudio del coste-beneficio no está perfectamente dilucidado.

\section{Bibliografía}

1. An, H.S., Evanich, C.J., Nowicki, B.H., Haughton, V.M.: Ideal thickness of Smith-Robinson graft for anterior cervical fusion. A cadaver study with computed tomographic correlation. Spine 1993; 18: 2043-2047.

2. Angevine, P.D., Arons, R.R., McCormick, P.C.: National and regional rates and variation of cervical discectomy with and without anterior fusion, 1990-1999. Spine 2003; 28 : 931-939.

3. Azmi, H., Schlenk, R.P.: Surgery for postarthrodesis 
adjacent-cervical segment degeneration. Neurosurg. Focus 2003; 15: article 6 .

4. Bailey, R., Badgley, C.: Stabilization of the cervical spine by anterior fusion. J. Bone Joint Surg. Am. 1960; 42: 565-594.

5. Casey, A.T.H.: Bone grafts and anterior cervical discectomy - lack of evidence, but no lack of opinion. Br. J. Neurosurg. 1999; 13: 445-448.

6. Cloward, R.B.: The anterior approach for removal of ruptured cervical discs. J. Neurosurg. 1958; 15: 602-617.

7. Comín, M., Prat, J. (eds): Biomecánica del raquis y sistemas de reparación. Valencia; IBV, 1995.

8. Dowd, G.C., Wirth, F.P.: Anterior cervical discectomy: is fusion necessary? J. Neurosurg. (Spine 1) 1999; 90: 8-12.

9. Greene, D.L., Crawford, N.R., Chamberlain, R.H., Crandall, D.: Biomechanical comparison of cervical interbody cage versus structural bone graft. Spine J. 2003; 3: 262-269.

10. Gercek, E., Arlet, V., Delisted, Marchesi, D.: Subsidence of stand-alone cervical cages in anterior interbody fusion: warning. Eur. Spine J. 2003; 12: 513-516.

11. Goffin, J., Van Calenbergh, F, Van Loon, J., Casey, A., Kehr, P., liebig, K., Lin, B., Logroscino, C., Sgrambiglia, R., Pointillart, V.: Intermediate follow-up after treatment of degenerative disc disease with the Bryan Cervical Disc Prosthesis: single-level and bi-level. Spine 2003; 28: 2673-2678.

12. González-Darder, J.M., Atienza, C.M., Molla, F.: Placa-caja para fijación cervical por vía anterior. Estudio biomecánico. Neurocirugía 2000; 11: 152-157.

13. González-Darder, J.M., Pesudo-Martínez, J.V., FeliuTatay, R.: Fusión cervical postdiscectomía. Estudio clínicoradiológico comparando el injerto óseo de cresta iliaca, placa cervical anterior con injerto óseo y placa-caja GD. Neurocirugía 2001; 12: 143-151.

14. Greene, D.L., Crawford, N.R., Chamberlain, R.H., Park, S.C., Crandall, D.: Biomechanical comparison of cervical interbody cage versus structural bone graft. Spine J. 2003; 3: 262-269.

15. Haid, R.W., Foley, K.T., Rodts, G.E., Barnes, B.: The Cervical Spine Study Group anterior cervical plate nomenclature. Neurosurg. Focus 2002; 12: article 15.

16. Hwang, Y.H., Kim. H.J., Lee, D.H., Lee, S.H., Kim, H.K., Kang, C.G., Chung, U.W.: Cervical plate cage system in degenerative cervical disease. J. Korean Neurosurg. Soc. 1999; 28: 1732-1737 (en coreano).

17. Jacobs, W., Anderson, P., Limbeek, J., Willems, P., Pavlov, P.: Single or double-level anterior interbody fusion techniques for cervical degenerative disc disease. Cochrane Database Syst. Rev. 2004; 18: CD004958.

18. Kandziora, F., Pflugmacher, R., Schafer, J., Born, C., Duda, G., Haas, N.P., Mittlmeier, T.: Biomechanical comparison of cervical spine interbody fusion cages. Spine 2001; 26 : 1850-1857.

19. Kher, P., Gosset, F., Graftiaux, A., Halbout, P.: Cervi- cal interbody fusion implant. Eur. J. Orthop. Surg. Traumatol. 1997; 7: 7-11.

20. Laing, R.J., Ng, I., Seeley, H.M., Hutchinson, P.J.: Prospective study of clinical and radiological outcome after anterior cervical discectomy. Br. J. Neurosurg. 2001; 15: 319323.

21. Ma, Y., Xi, J., Chen, X., Guan, C., Quan, C.: Insertion of PCB to treat traumatic cervical intervertebral disc herniation.: Chin. J. Traumatol. 2002; 5: 267-270.

22. Martín, R., Carda, J.R., Pint, J.I., Sanz, F., Montiaga, F., Paternina, B., Triguero, F., Izquierdo, J.M., VázquezBarquero, A.: Disectomía cervical y artrodesis intersomática mediante técnica de Cloward: Análisis retrospectivo de las complicaciones y los resultados radiológicos en 167 casos. Neurocirugia 2002; 13: 265-284.

23. Martínez Ruíz, F.: Biomecánica de la columna vertebral y sus implantes. Madrid, Nueva Imprenta, 1992.

24. Matge, G.: Cervical cage fusion with 5 different implants: 250 cases. Acta Neurochir. (Wien); 144: 539-549.

25. Orozco, R., Llovet, R.: Osteosíntesis en las fracturas del raquis cervical. Rev. Ortop. Traumatol. 1970; 14: 285288.

26. Pflugmacher, R., Schleicher, P., Gumnior, S., Turan, O., Scholz, M., Eindorf, T., Haas, N.P., Kandziora, F.: Biomechanical comparison of bioabsorbable cervical spine interbody fusion cages. Spine 2004; 29: 1717-1722.

27. Porras-Estrada, L.F., Ugarriza-Echevarrieta, L.F., Lorenzana-Honrado, L., Rodriguez-Sánchez, L.A., GarcíaYagüe, L.M., Fernández-Portales, I., Gómez-Perals, L., Cabezudo, J.M.: Estudio comparativo de la artrodesis cervical con injerto óseo roscado e implante de titanio. Neurocirugía 2004; 15: $270-278$.

28. Reitman, C.A., Hipp, J.A., Nguyen, L., Esses, S.I.: Changes in segmental intervertebral motion adjacent to cervical arthrodesis: a prospective study. Spine 2994; 29: E221226.

29. Samandouras, G., Shafafy, M., Hamlyn, P.J.: A new anterior cervical instrumentation system combining an intradiscal cage with an integrated plate: an early technical report.: Spine 2001; 26: 1188-1192 16

30. Samartzis, D., Shen, F.H., Lyon, C., Phillips, M., Goldberg, E.J., An, H.S.: Does rigid instrumentation increase the fusion rate in one-level anterior cervical discectomy and fusion? Spine J. 2004; 4: 636-643.

31. Savolainen,S., Rinne,J., Hernesniemi,J.: A prospective randomized study of anterior single-level cervical disc operations with long-term follow-up: Surgical fusion is unnecessary. Neurosurg. 1998; 43:51-55.

32. Schlenk, R.P., Steward, T., Benzel, E.C.: The biomechanics of iatrogenic spinal destabilization and implant failure. Neurosurg. Focus 2002; 15: article 2.

33. Schulte, K., Clark, C.R., Goel, V.K.: Kinematics of the 
cervical spine following discectomy and stabilization. Spine 1989; 14: 1116-1121.

34. Smith, G.W., Robinson, R.A.: The treatment of certain cervical spine disorders by anterior removal of the intervertebral disc and interbody fusion. J. Bone Joint Surg. Am. 1958; 40: 607-624.

35. Thome, C., Krauss, J.K., Zevgaridis, D.: A prospective clinical comparison of rectangular titanium cages and iliac crest autografts in anterior discectomy and fusion. Neurosurg. Rev. 2004; 27: 34-41.

36. Tureyen, K.: Disc height loss after anterior cervical microdiscectomy with titanium intervertebral cage fusion. Acta Neurochir. (Wien) 2003; 145: 565-569.

37. Zdeblick, T.A., Phillips, F.M.: Interbody cage devices. Spine 2003; 28 (Suppl 15): S2-7.

González-Darder, J.M.: Evolución de la artrodesis cervical postdiscectomía: injerto óseo, placa, caja intersomática y placa-caja. Neurocirugía 2006; 17: 140-147.

Correspondencia postal: Dr. José M. González Darder. Servicio de Neurocirugía. Hospital General de Castellón. 12003 Castellón

\section{Comentario al trabajo: Evolución de la artrodesis cervical postdiscectomía: injerto óseo, placa, caja intersomática y placa-caja de J.M. González Darder.}

El autor hace una descripción exhaustiva de la evolución de la técnica quirúrgica para el tratamiento de la patología espinal cervical. Es verdaderamente espectacular cómo se han desarrollado diferentes materiales y sistemas dirigidos a conseguir una mayor estabilidad biomecánica, una mayor tasa de fusión y una menor degeneración posterior de los espacios vecinos al segmento espinal fusionado. Sin embargo, las ventajas de gran parte de estos "adelantos" se han basado sólo en razones biomecánicas teóricas o en modelos biomecánicos en cadáveres. Una pregunta importante que cabe hacerse es: ¿Han contribuido realmente estos avances tecnológicos a mejorar el pronóstico de los pacientes operados de patología cervical?, y en caso afirmativo ¿Cuál es la relación coste-beneficio? Como el propio autor comenta, Jacobs y cols ${ }^{4}$ en la revisión sistemática para la Cochrane ya alertan sobre la pobre calidad de los estudios publicados sobre el tratamiento quirúrgico de la patología cervical degenerativa, no encontrando diferencias significativas respecto a la evolución de los pacientes dependiendo de la técnica quirúrgica empleada (si acaso a favor de la discectomía simple). Es más, desde un punto de vista estricto, según la medicina basada en la evidencia, ni siquiera hay certeza de que los pacientes con mielo-radiculopatía cervical espondilótica se beneficien del tratamiento quirúrgico ${ }^{3}$. Fouyas y cols en otra revisión para la Cochrane encontraron sólo una pequeña ventaja a corto plazo del tratamiento quirúrgico frente al tratamiento conservador en pacientes con mielo-radiculopatía cervical espondilótica ${ }^{3}$.

El autor también comenta el importante aumento de las intervenciones cervicales observadas en estudios epidemiológicos en Estados Unidos. Un hecho similar ha sido observado respecto a la patología degenerativa lumbar, y un análisis de sus posibles causas han sido analizados recientemente por Deyo y cols ${ }^{2}$. Sin embargo, en la revisión sistemática de la literatura llevada a cabo por Bono y cols ${ }^{1}$ no se encontró diferencia significativa en el pronóstico de los pacientes intervenidos de patología lumbar degenerativa en la decada de los 80 frente a los operados en los 90.

Lo que sí parece evidente es que se precisan estudios randomizados de calidad que confirmen la supuestas ventajas de estos nuevos materiales y sistemas.

\section{Bibliografía}

1. Bono, C.M., Lee, C.K.: Critical analysis of trends in fusion for degenerative disc disease over the past 20 years: influence of technique on fusion rate and clinical outcome. Spine 2004; 29: 455-463.

2. Deyo, R.A., Nachemson, A., Mirza, S.K.: Spinal-fusion surgery - the case for restraint. N Engl J Med 2004; 350: 722726

3. Fouyas, I.P., Statham, P.F., Sandercock, P.A.: Cochrane review on the role of surgery in cervical spondylotic radiculomyelopathy. Spine 2002; 27: 736-747.

4. Jacobs, W., Anderson, P., Limbeek, J., Pavlov, P.: Single or double-level anterior interbody fusion techniques for cervical degenerative disc disease. Cochrane Database Syst. Rev. 2004; 18: CD004958.

$$
\text { R. Alday }
$$

Madrid 